\title{
Using the Reverse Shock Index at the Injury Scene and in the Emergency Department to Identify High-Risk Patients: A Cross-Sectional Retrospective Study
}

\author{
Wei-Hung Lai ${ }^{1, \dagger}{ }^{,}$Cheng-Shyuan Rau ${ }^{2, \dagger}{ }^{\dagger}$, Shiun-Yuan Hsu ${ }^{1}$, Shao-Chun $\mathrm{Wu}^{3}$, Pao-Jen Kuo ${ }^{4}$, \\ Hsiao-Yun Hsieh ${ }^{1}$, Yi-Chun Chen ${ }^{1}$ and Ching-Hua Hsieh ${ }^{3, *}$ \\ 1 Department of Trauma Surgery, Kaohsiung Chang Gung Memorial Hospital and Chang Gung University \\ College of Medicine, Kaohsiung 833, Taiwan; abdiel@cgmh.org.tw (W.-H.L.); ah.lucy@hotmail.com (S.-Y.H.); \\ sylvia19870714@hotmail.com (H.-Y.H.); libe320@yahoo.com.tw (Y.-C.H.) \\ 2 Department of Neurosurgery, Kaohsiung Chang Gung Memorial Hospital and Chang Gung University \\ College of Medicine, Kaohsiung 833, Taiwan; ersh2127@cloud.cgmh.org.tw \\ 3 Department of Anesthesiology, Kaohsiung Chang Gung Memorial Hospital and Chang Gung University \\ College of Medicine, Kaohsiung 833, Taiwan; shaochunwu@gmail.com \\ 4 Department of Plastic and Reconstructive Surgery, Kaohsiung Chang Gung Memorial Hospital and Chang \\ Gung University College of Medicine, Kaohsiung 833, Taiwan; bow110470@gmail.com \\ * Correspondence: m93chinghua@gmail.com; Tel.: +886-7-732-7476; Fax: +886-7-345-0431 \\ + Indicates equal contribution in authorship.
}

Academic Editor: Paul B. Tchounwou

Received: 28 January 2016; Accepted: 21 March 2016; Published: 24 March 2016

\begin{abstract}
Background: The ratio of systolic blood pressure (SBP) to heart rate (HR), called the reverse shock index (RSI), is used to evaluate the hemodynamic stability of trauma patients. A SBP lower than the HR $(\mathrm{RSI}<1)$ indicates the probability of hemodynamic shock. The objective of this study was to evaluate whether the RSI as evaluated by emergency medical services (EMS) personnel at the injury scene (EMS RSI) and the physician in the emergency department (ED RSI) could be used as an additional variable to identify patients who are at high risk of more severe injury. Methods: Data obtained from all 16,548 patients added to the trauma registry system at a Level I trauma center between January 2009 and December 2013 were retrospectively reviewed. Only patients transferred by EMS were included in this study. A total of 3715 trauma patients were enrolled and subsequently divided into four groups: group I patients had an EMS RSI $\geqslant 1$ and an ED RSI $\geqslant 1(n=3485)$; group II an EMS RSI $\geqslant 1$ and an ED RSI $<1(n=85)$; group III an EMS RSI $<1$ and an ED RSI $\geqslant 1(n=98)$; and group IV an EMS RSI $<1$ and a ED RSI $<1(n=47)$. A Pearson's $\chi^{2}$ test, Fisher's exact test, or independent Student's t-test was conducted to compare trauma patients in groups II, III, and IV with those in group I. Results: Group II and IV patients had a higher injury severity score, a higher incidence of commonly associated injuries, and underwent more procedures (including intubation, chest tube insertion, and blood transfusion in the ED) than patients in group I. Group II and IV patients were also more likely to receive a severe injury to the thoracoabdominal area. These patients also had worse outcomes regarding the length of stay in hospital and intensive care unit (ICU), the proportion of patients admitted to ICU, and in-hospital mortality. Group II patients had a higher adjusted odds ratio for mortality (5.8-times greater) than group I patients. Conclusions: Using an RSI $<1$ as a threshold to evaluate the hemodynamic condition of the patients at the injury scene and upon arrival to the ED provides valid information regarding deteriorating outcomes for certain subgroups of patients in the ED setting. Particular attention and additional resources should be provided to patients with an EMS RSI $\geqslant 1$ that deteriorates to an RSI $<1$ upon arrival to the ED since a higher odds of mortality was found in these patients.
\end{abstract}


Keywords: reverse shock index (RSI); shock index (SI); emergency medical services (EMS); injury severity score (ISS); length of stay (LOS); mortality

\section{Background}

Acute trauma patients presenting with shock in the prehospital or emergency department (ED) setting need focused care. Measurement of vital signs, including systolic blood pressure (SBP), is often included in the initial triage for acute trauma patients and is recommended by many clinical guidelines as a basic part of the initial assessment of circulatory blood volume by emergency medical services (EMS) personnel. In addition to the trauma cause, a description of the hemodynamic status, including its severity, provided by responsible EMS personnel could be an important reference for the emergency physician, who could use this information to provide adequate patient care [1,2].

Hypovolemic shock is the most common type of shock in patients who experience traumatic injury. In a meta-analysis of six observational studies, the prevalence of hypotensive shock in the prehospital setting was between 9.5 and 19 per 1000 EMS contacts with an in-hospital shock mortality between $33 \%$ and $52 \%$ [3]. In addition, the prevalence of hypotension in the ED was 4-13/1000 ED contacts with a mortality of $12 \%$ [3]. To identify hypovolemic shock, isolated vital signs, such as SBP and heart rate (HR), have been shown to be unreliable [4,5]. For example, individuals who are habitually hypertensive can have a normal blood pressure during shock, and hypotensive individuals can have normal tissue perfusion [6,7]. Some observational studies conducted in the ED and prehospital setting with hypotensive patients who experienced a trauma have advocated for a higher SBP threshold to correspond with the actual mortality rate [8-10]. The shock index (SI), the ratio of HR to SBP, has been assessed as a marker of significant injury in trauma patients with hypovolemic shock [11-13], and been found to be more useful in predicting early shock than either the HR or the SBP alone and to correlate with other indices of end-organ perfusion (such as central venous oxygen saturation and arterial lactic acid concentration) [14]. The SI has been demonstrated to be a capable measure for hemodynamic instability [15-17] and a useful guide for diagnosing early acute hypovolemia in the presence of normal HR and blood pressure [18]. It has also been shown to be a clinical indicator of hypovolemic shock upon arrival to the ED with respect to transfusion requirements and hemostatic resuscitation [19].

An elevated SI $>0.7$ is correlated with reduced left ventricular end-diastolic pressure [20] and reductions in circulatory blood volume [13] even when the pulse rate and SBP remain in the normal range. A pre-intubation SI greater than or equal to 0.8 is an independent risk factor for cardiovascular deterioration after emergency intubation in the ED [21]. An SI that exceeds 0.9 between the field and the ED may predict higher mortality [13]. The sensitivity and specificity of an SI greater than 0.9 as a predictor of massive transfusion (defined as 24 -h red blood cell transfusion $\geqslant 9$ units) are $63 \%$ and $83 \%$, respectively [22]. An SI greater than or equal to 1 is associated with an adjusted odds ratio (AOR) of 10.5 (95\% confidence interval (CI): 9.3-11.7) for 30-day mortality [23]. In addition, patients with an SI $\geqslant 1.0$, despite prehospital crystalloid resuscitation, had a significantly higher transfusion requirement and a higher mortality rate than other major trauma patients [15]. Furthermore, an SI greater than 1.4 has been proposed as a more practical cutoff for predicting massive transfusion in trauma patients [22].

Although the SI is a very practical and useful predictor of outcomes for trauma patients, the literature has shown that the relevant SI cut-off point varies depending on the cause of trauma $[12,13,20,23,24]$ and patient's illness [23]. Moreover, the calculation of the SI as the ratio of HR to SBP appears contradictory to the basic concept of shock, which is generally thought of as an unstable hemodynamic status in which the SBP is lower than the HR, and not as the SI, which indicates that the HR is higher than the SBP. Therefore, the reverse shock index (RSI), the ratio of SBP to HR, is often preferred to evaluate the hemodynamic stability of trauma patients. An RSI $<1$ indicates that the 
SBP is lower than the HR, and implies that the patient is probably in shock. Moreover, the RSI could be assessed without any additional calculation or equipment by first responders upon arrival at the site of injury or to the ED.

Identification of patients with shock is crucial as prompt treatment improves the prognosis and the optimal treatment differs depending on the cause [7]. The objective of this study was to evaluate whether an RSI evaluated by EMS personnel at the scene of injury (EMS RSI) and the physician at the ED (ED RSI) could be used to identify patients who are at high risk for more severe complications. To accomplish this, we used data from a trauma registry system collected over a five-year period at a Level I trauma center.

\section{Methods}

\subsection{Ethics Statement}

The hospital's institutional review board (IRB) approved this study (approval number 104-0578B). Informed consent was waived according to the IRB regulations.

\subsection{Study Design}

This retrospective study was conducted at the Kaohsiung Chang Gung Memorial Hospital, a 2400-bed facility and Level I regional trauma center that provides care to trauma patients primarily from southern Taiwan. This study reviewed all 16,548 hospitalized and registered patients added to the Trauma Registry System between 1 January 2009 and 31 December 2013 (Figure 1). During this time, only patients who were transferred by EMS were included in this study. Those who were transferred from other hospitals or arrived by private vehicles were not included in the study population. Patients who had incomplete data were also excluded. The RSI was calculated as the ratio of SBP to HR $(\mathrm{RSI}=\mathrm{SBP} / \mathrm{HR})$. In total, 3715 trauma patients were enrolled in this study. These patients were then divided into 4 groups. Group I included those with an EMS RSI $\geqslant 1$ and an ED RSI $\geqslant 1(n=3485)$; group II included those with an EMS RSI $\geqslant 1$ and an ED RSI $<1(n=85)$; group III those with an EMS RSI $<1$ and an ED RSI $\geqslant 1(n=98)$; and group IV those with an EMS RSI $<1$ and an ED RSI $<1$ $(n=47)$. Group I (patients with a stable hemodynamic status at the injury scene and in the ED) was used as a reference for comparison with group II (patients with a stable hemodynamic status at the injury scene but who got worse upon arrival to the ED), group III (patients with hemodynamic instability at the injury scene but who improved upon arrival to the ED), and group IV (patients with hemodynamic instability at the injury scene and in the ED). The vital signs of the patients generally would be measured within 5 min upon arrival to the ED.

Detailed patient information regarding age, sex, vital signs (assessed by the EMS personnel at the injury scene and by the physician upon arrival to the ED), the Glasgow coma scale (GCS) score (assessed upon arrival to the ED), procedures performed by EMS personnel at the injury scene (intubation, airway placement, neck collar placement, backboard or spinal immobilization, oxygenation, and cardiopulmonary resuscitation) and by the physician in the ED (intubation, chest tube insertion, and blood transfusion), the abbreviated injury scale (AIS) score for each body region, the injury severity score (ISS), the new injury severity score (NISS), the trauma and injury severity score (TRISS), length of stay (LOS) in the hospital, LOS in the intensive care unit (ICU), in-hospital mortality, and complications associated with injuries. In our study, the primary outcomes were in-hospital mortality and injury severity as measured by different scoring systems (GCS, AIS, ISS, NISS, and TRISS). The secondary outcomes were LOS in the hospital and in the ICU.

Data were compared using SPSS version 20 statistical software (IBM Corporation, Armonk, NY, USA). We used a Pearson's $\chi^{2}$ test, Fisher's exact test, or independent Student's $t$-test, as applicable. The Mann Whitney U test was used to compare the LOS in hospital and ICU. All results are presented as a mean \pm standard error. A $p$-value of $<0.05$ was considered as statistically significant. Odds ratios 
(ORs) were calculated with 95\% confidence intervals (CIs). AORs for mortality adjusted by the ISS with 95\% CIs were also calculated.

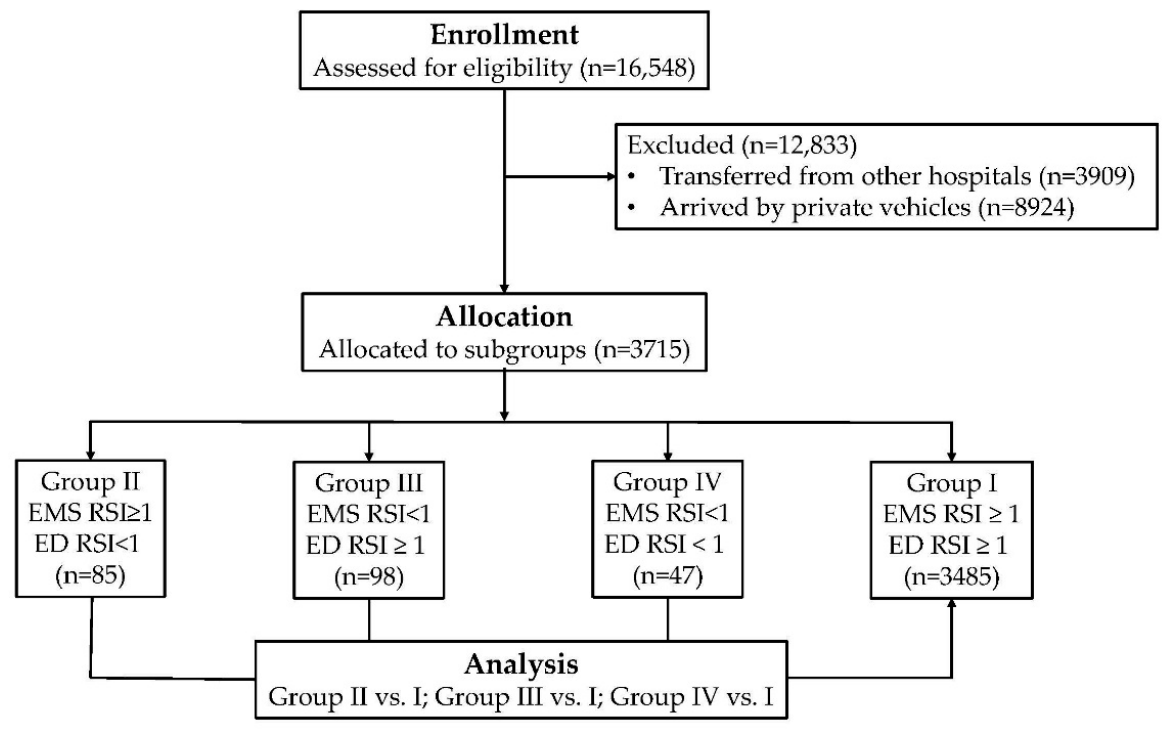

Figure 1. Flow diagram of studied groups of patients.

\section{Results}

\subsection{Patient Injury Characteristics}

The mean age of patients in groups I, II, III, and IV were $41.9 \pm 14.4,36.6 \pm 13.5,39.2 \pm 15.5$, and $38.9 \pm 12.5$ years, respectively (Table 1$)$. Compared to patients in group I, patients in group II were younger $(p=0.001)$, and statistically significant differences regarding sex were found between group II patients (men: $n=60(70.6 \%)$; women: $n=25(29.4 \%)$ ) and group IV patients (men: $n=36(76.6 \%)$; women: $n=11(23.4 \%)$ ) when compared to group I patients (men: $n=2026(58.1 \%)$; women: $n=1459$ $(41.9 \%))$. There were also significantly lower GCS scores in both group II (12.4 \pm 3.8$)$ and group IV $(13.0 \pm 3.3)$ than in group I $(14.3 \pm 2.1)$. In addition, the distribution of GCS scores $(\leqslant 8,9-12$, or $\geqslant 13)$ were different in group II and IV patients than group I patients. The analysis of AIS scores revealed that group II patients sustained significantly higher rates of injuries to the head/neck, thorax, and abdomen, but lower rates of injuries to the extremities than group I patients; group III patients sustained significantly higher rates of injuries to the thorax; and group IV patients sustained significantly higher rates of injuries to the head/neck, thorax, and abdomen. A significantly higher ISS was found in group II (15.1 $\pm 11.1 ; p<0.001)$, group III $(10.5 \pm 7.1 ; p=0.026)$, and group IV $(15.8 \pm 11.3 ; p<0.001)$ in comparison to group I $(8.9 \pm 6.9)$. When stratified by ISS $(<16,16-24$, or $\geqslant 25)$, group II included more patients with an ISS $\geqslant 25$ and less patients with an ISS $<16$ than in group I while group IV included more patients with an ISS $\geqslant 25$ and an ISS between 16 and 24 and less patients with an ISS of $<16$ than group I. No differences were found between group III and group I regardless of the stratification by ISS $(<16,16-24$, or $\geqslant 25)$. Likewise, we also found a significantly higher NISS in group II (17.8 \pm 14.4 ; $p<0.001)$, group III $(12.3 \pm 8.3 ; p=0.036)$, and group IV $(16.7 \pm 12.6 ; p<0.001)$ than in group I $(10.5 \pm 8.5)$. When compared with the TRISS of group I $(0.991 \pm 0.093)$, a significantly lower TRISS, indicating a lower survival rate, was found in group II $(0.953 \pm 0.213 ; p<0.001)$ and group IV $(0.957 \pm 0.204 ; p<0.001)$, but not in group III $(0.980 \pm 0.142 ; p=0.233)$. The in-hospital mortality rates for group I, II, III, and IV were $0.9 \%, 12.9 \%, 1.0 \%$, and $4.3 \%$, respectively. After adjusting for ISS, the AOR for mortality for patients in group II (AOR = 5.8, 95\% CI: 2.3-14.4, $p<0.001)$ and IV (AOR = 1.1, $95 \%$ CI: $1.1-1.1, p<0.001)$ was significantly greater than that of patients in group I. No difference in the AOR for mortality was found between group III and I. 
Table 1. Demographic and injury characteristics of hospitalized trauma patients.

\begin{tabular}{|c|c|c|c|c|c|c|c|c|c|c|}
\hline \multirow{2}{*}{ Variables } & \multirow{2}{*}{$\begin{array}{l}\text { EMS RSI } \geqslant 1 \text { ED } \\
\text { RSI }<1 n=85 \text { (II) }\end{array}$} & \multirow{2}{*}{$\begin{array}{c}\text { EMS RSI }<1 \text { ED RSI } \\
\geqslant 1 n=98 \text { (III) }\end{array}$} & \multirow{2}{*}{$\begin{array}{c}\text { EMS RSI }<1 \text { ED } \\
\text { RSI }<1 n=47 \text { (IV) }\end{array}$} & \multirow{2}{*}{$\begin{array}{c}\text { EMS RSI } \geqslant 1 \text { ED RSI } \\
\geqslant 1 n=3485 \text { (I) }\end{array}$} & \multicolumn{2}{|c|}{ OR $(95 \% C I) p$} & \multicolumn{2}{|c|}{ OR $(95 \% C I) p$} & \multicolumn{2}{|c|}{ OR $(95 \% C I) p$} \\
\hline & & & & & \multicolumn{2}{|c|}{ II $v s$. I } & \multicolumn{2}{|c|}{ III vs. I } & \multicolumn{2}{|c|}{ IV $v s$. I } \\
\hline Age & $36.6 \pm 13.5$ & $39.2 \pm 15.5$ & $38.9 \pm 12.5$ & $41.9 \pm 14.4$ & - & 0.001 & - & 0.071 & - & 0.158 \\
\hline Gender & $60(706)$ & $55(561)$ & $36(766)$ & $2026(581)$ & $17(11-28)$ & 0.021 & $09(06-14)$ & 0.690 & & 0.011 \\
\hline $\begin{array}{l}\text { Male } \\
\text { Female }\end{array}$ & $25(29.4)$ & $43(43.9)$ & $11(23.4)$ & $1459(41.9)$ & $0.6(0.4-0.9)$ & & $\begin{array}{l}0.9(0.6-1.4) \\
1.1(0.7-1.6)\end{array}$ & & $\begin{array}{l}2.4(1.2-4.7) \\
0.4(0.2-0.8)\end{array}$ & \\
\hline$\leqslant 8$ & $16(18.8)$ & $5(5.1)$ & $7(14.9)$ & $148(4.2)$ & $5.2(3.0-9.2)$ & $<0.001$ & $1.2(0.5-3.0)$ & 0.680 & $3.9(1.7-9.0)$ & $<0.00$ \\
\hline 9-12 & $13(15.3)$ & $5(5.1)$ & $5(10.6)$ & $156(4.5)$ & $3.9(2.1-7.1)$ & $<0.001$ & $1.1(0.5-2.9)$ & 0.768 & $2.5(1.0-6.5)$ & 0.044 \\
\hline$\geqslant 13$ & $56(65.9)$ & $88(89.8)$ & $35(74.5)$ & $3181(91.3)$ & $0.2(0.1-0.3)$ & $<0.001$ & $0.8(0.4-1.6)$ & 0.609 & $0.3(0.1-0.5)$ & $<0.00$ \\
\hline Head/Neck & $39(45.9)$ & $32(32.7)$ & $22(46.8)$ & $1074(30.8)$ & $1.9(1.2-2.9)$ & 0.003 & $1.1(0.7-1.7)$ & 0.698 & $2.0(1.1-3.5)$ & 0.019 \\
\hline Face & $19(22.4)$ & $21(21.4)$ & $11(23.4)$ & 734 ( (21.1) & $1.1(0.6-1.8)$ & 0.773 & $1.0(0.6-1.7)$ & 0.930 & $1.1(0.6-2.3)$ & 0.696 \\
\hline Thorax & $29(34.1)$ & $23(23.5)$ & $23(48.9)$ & 477 (13.7) & $3.3(2.1-5.2)$ & $<0.001$ & $1.9(1.2-3.1)$ & 0.006 & $6.0(3.4-10.8)$ & $<0.00$ \\
\hline Abdomen & $21(24.7)$ & $10(10.2)$ & $19(40.4)$ & $237(6.8)$ & $4.5(2.7-7.5)$ & $<0.001$ & $1.6(0.8-3.0)$ & 0.190 & $9.3(5.1-16.9)$ & $<0.001$ \\
\hline Extremity & $54(63.5)$ & $72(73.5)$ & $33(70.2)$ & 2585 (74.2) & $0.6(0.4-1.0)$ & 0.027 & $1.0(0.6-1.5)$ & 0.875 & $0.8(0.4-1.5)$ & 0.538 \\
\hline ISS & $15.1 \pm 11.1$ & $10.5 \pm 7.1$ & $15.8 \pm 11.3$ & $8.9 \pm 6.9$ & & $<0.001$ & & 0.026 & & $<0.001$ \\
\hline$<16$ & $48(56.5)$ & $76(77.6)$ & $23(48.9)$ & $2794(80.2)$ & $0.3(0.2-0.5)$ & $<0.001$ & $0.9(0.5-1.4)$ & 0.522 & $0.2(0.1-0.4)$ & $<0.00$ \\
\hline $16-24$ & $16(18.9)$ & $16(6.1)$ & $14(29.8)$ & $483(13.9)$ & $1.4(0.8-2.5)$ & 0.192 & $1.2(0.7-2.1)$ & 0.487 & $2.6(1.4-5.0)$ & 0.002 \\
\hline $\begin{array}{l}\geqslant 25 \\
\text { NISS }\end{array}$ & $\begin{array}{l}21(24.7) \\
178+144\end{array}$ & $\begin{array}{c}6(6.1) \\
12+83\end{array}$ & $10(21.3)$ & 208 (6.0) & $5.2(3.1-8.6)$ & $<0.001$ & $1.0(0.5-2.4)$ & 0.949 & $4.3(2.1-8.7)$ & $<0.00$ \\
\hline $\begin{array}{l}\text { NISS } \\
\text { TRISS }\end{array}$ & $\begin{array}{l}17.8 \pm 14.4 \\
0.953+0.213\end{array}$ & $\begin{array}{c}12.3 \pm 8.3 \\
0980 \pm 0142\end{array}$ & $\begin{array}{l}16.7 \pm 12.6 \\
0.957 \pm 0.64\end{array}$ & $\begin{array}{c}10.5 \pm 8.5 \\
0.991+0.093\end{array}$ & - & $<0.001$ & - & 0.036 & - & $<0.001$ \\
\hline $\begin{array}{l}\text { TRISS } \\
\text { Mortality }\end{array}$ & $\begin{array}{c}0.953 \pm 0.213 \\
11(12.9)\end{array}$ & $\begin{array}{l}0.980 \pm 0.142 \\
1(1.0)\end{array}$ & $\begin{array}{c}0.957 \pm 0.204 \\
2(4.3)\end{array}$ & $\begin{array}{c}0.991 \pm 0.093 \\
32(0.9)\end{array}$ & $16.0(7.8-33.0)$ & $\begin{array}{l}<0.001 \\
<0.001\end{array}$ & $1.1(0.2-8.2)$ & $\begin{array}{l}0.233 \\
0.917\end{array}$ & $4.8(1.1-20.6)$ & $\begin{array}{l}0.016 \\
0.020\end{array}$ \\
\hline ISS & & & & & $5.8(2.3-14.4)$ & $<0.001$ & $1.0(1.0-1.1)$ & 0.053 & $1.1(1.1-1.1)$ & $<0.001$ \\
\hline
\end{tabular}




\subsection{Management Characteristics}

There were no significant differences regarding the transport times for group II, III, and IV compared to group I (Table 2). Regarding the procedures performed by the EMS personnel, more group II patients underwent airway placement, placement of a neck collar, backboard and spinal immobilization, an oxygen supplement, and cardiopulmonary resuscitation than group I patients; more group III patients underwent airway placement, backboard immobilization, and an oxygen supplement; and more group IV patients underwent placement of a neck collar, backboard immobilization, and an oxygen supplement. Regarding the procedures performed in the ED, more group II and group IV patients underwent intubation, chest tube insertion, and blood transfusion than group I patients. No significant differences regarding the procedures performed in the ED were noted between group III and I patients.

\subsection{Associated Injuries}

As shown in the Table 3, the OR for sustaining intracerebral hematoma (OR: 3.8, 95\% CI: 1.6-8.9, $p=0.001$ ), a cervical vertebral fracture (OR: 4.5, 95\% CI: 1.4-15.2, $p=0.007$ ), pneumothorax (OR: 3.0, 95\% CI: 1.1-8.4, $p=0.031$ ), hemopneumothorax (OR: 6.2, 95\% CI: 2.4-16.1, $p<0.001$ ), a lung contusion (OR: 4.0, 95\% CI: 1.4-11.3, $p=0.006$ ), hepatic injury (OR: 8.0, 95\% CI: 4.2-15.4, $p<0.001$ ), splenic injury (OR: 6.1, 95\% CI: 2.1-17.8, $p<0.001$ ), retroperitoneal injury (OR: 16.8, 95\% CI: 3.2-87.7, $p<0.001$ ), and renal injury (OR: 6.4, 95\% CI: 1.4-29.0, $p=0.005$ ) was statistically significantly higher for group II patients than group I patients. Similarly, the OR for sustaining a rib fracture (OR: 1.8, 95\% CI: 1.1-3.1, $p=0.023$ ), hemothorax (OR: 4.7, 95\% CI: 2.0-11.42, $p<0.001$ ), and hepatic injury (OR: 2.6, 95\% CI: $1.0-6.7, p=0.035$ ) was statistically significantly higher for group III patients than group I patients. Group IV patients had a statistically significantly higher OR for sustaining a rib fracture (OR: 3.7, 95\% CI: 2.0-7.0, $p<0.001$ ), hemothorax (OR: 6.7, 95\% CI: 2.3-19.3, $p<0.001$ ), pneumothorax (OR: 4.1, 95\% CI: $1.2-13.6, p=0.012)$, hemopneumothorax (OR: 14.4, 95\% CI: 5.8-36.2, $p<0.001$ ), an intra-abdominal injury (OR: 7.7, 95\% CI: 3.4-17.8, $p<0.001$ ), hepatic injury (OR: 8.5, 95\% CI: 3.7-19.7, $p<0.001$ ), splenic injury (OR: 11.5, 95\% CI: 3.9-34.2, $p<0.001$ ), and pelvic fracture (OR: 4.9, 95\% CI: 2.1-11.1, $p<0.001$ ) than group I patients.

\subsection{LOS in Hospital and ICU}

A significantly longer hospital LOS was found among group II and IV patients compared with group I patients (15.2 and 16.9 vs. 9.5 days, respectively; $p<0.001$ ) (Table 4). Likewise, a significantly larger proportion of group II and IV patients than group I patients were admitted to the ICU (45.9\% and $51.1 \%$ vs. $16.5 \%$, respectively; $p<0.001)$, but the ICU LOS was not significantly longer (13.1 and 13.0 vs. 7.6 days, respectively; $p=0.296$ and $p=0.070$, respectively).

\section{Discussion}

In this study, group II patients (i.e., those who had a stable hemodynamic status as evaluated by EMS personnel at the injury scene but got worse upon arrival to the ED) and group IV patients (i.e., those who had an unstable hemodynamic condition both at the injury scene and upon arrival to the ED) had worse outcomes than group I patients (i.e., those who had a stable hemodynamic condition at the injury scene and in the ED). Group II and IV patients had a higher ISS, a higher incidence of commonly associated injuries, and underwent more procedures than group I patients. These patients also had worse outcomes regarding hospital LOS, the proportion of patients admitted to the ICU, and in-hospital mortality. On the other hand, group III patients (i.e., those who had an improved hemodynamic condition upon arrival to the ED than that at the injury scene), had a good response after EMS involvement and demonstrated no significant differences regarding injury severity, mortality, the proportion of patients admitted to the ICU, and hospital and ICU LOS when compared to group I patients. 
Table 2. Transport time and procedures performed by EMS and ED personnel.

\begin{tabular}{|c|c|c|c|c|c|c|c|c|c|c|}
\hline \multirow[t]{2}{*}{ Variables } & \multirow{2}{*}{$\begin{array}{c}\text { EMS RSI } \geqslant 1 \text { ED } \\
\text { RSI }<1 \\
n=85 \text { (II) }\end{array}$} & \multirow{2}{*}{$\begin{array}{c}\text { EMS RSI }<1 \text { ED } \\
\text { RSI } \geq 1 \\
n=98 \text { (III) }\end{array}$} & \multirow{2}{*}{$\begin{array}{c}\text { EMS RSI }<1 \text { ED } \\
\text { RSI }<1 \\
n=47 \text { (IV) }\end{array}$} & \multirow{2}{*}{$\begin{array}{c}\text { EMS RSI } \geqslant 1 \text { ED } \\
\text { RSI } \geqslant 1 \\
n=3485 \text { (I) }\end{array}$} & \multicolumn{2}{|c|}{ OR $(95 \% C I) p$} & \multicolumn{2}{|c|}{ OR $(95 \% C I) p$} & \multicolumn{2}{|c|}{ OR $(95 \% C I) p$} \\
\hline & & & & & \multicolumn{2}{|c|}{ II $v s$. I } & \multicolumn{2}{|c|}{ III $v s$. I } & \multicolumn{2}{|c|}{ IV vs. I } \\
\hline Mean (mins) & $23.0 \pm 9.6$ & $24.2 \pm 8.9$ & $22.1 \pm 6.7$ & $22.8 \pm 9.4$ & - & 0.864 & - & 0.138 & - & 0.603 \\
\hline Range (mins) & $10-67$ & $12-68$ & $10-36$ & $4-142$ & - & - & - & - & - & - \\
\hline \multicolumn{11}{|l|}{$\begin{array}{l}\text { Procedures performed } \\
\text { by EMS personnel }\end{array}$} \\
\hline Intubation & $0(0.0)$ & $0(0.0)$ & $0(0.0)$ & $0(0.0)$ & - & - & - & - & - & - \\
\hline Airway & $2(2.4)$ & $4(4.1)$ & $1(2.1)$ & $17(0.5)$ & $4.9(1.1-21.6)$ & 0.020 & $8.7(2.9-26.3)$ & $<0.001$ & $4.4(0.6-34.0)$ & 0.117 \\
\hline Neck collar & $29(34.1)$ & $28(28.6)$ & $22(46.8)$ & 849 (24.4) & $1.6(1.0-2.5)$ & 0.039 & $1.2(0.8-1.9)$ & 0.339 & $2.7(1.5-4.9)$ & $<0.001$ \\
\hline Backboard & $36(42.4)$ & $36(36.7)$ & $23(48.9)$ & $930(26.7)$ & $2.0(1.3-3.1)$ & 0.001 & $1.6(1.1-2.4)$ & 0.027 & $2.6(1.5-4.7)$ & 0.001 \\
\hline Spinal immobilizer & $3(3.5)$ & $1(1.0)$ & $0(0.0)$ & $12(0.3)$ & $10.6(2.9-38.2)$ & $<0.001$ & $3.0(0.4-23.2)$ & 0.272 & $1.0(1.0-1.0)$ & 0.687 \\
\hline Oxygenation & $15(17.6)$ & $12(12.2)$ & $11(23.4)$ & $203(5.8)$ & $3.5(2.0-6.2)$ & $<0.001$ & $2.3(1.2-4.2)$ & 0.008 & $4.9(2.5-9.9)$ & $<0.001$ \\
\hline $\begin{array}{l}\text { Cardiopulmonary } \\
\text { resuscitation }\end{array}$ & $1(1.2)$ & $0(0.0)$ & $0(0.0)$ & $1(0.0)$ & $41.5(2.6-668.7)$ & $<0.001$ & - & 0.867 & - & 0.908 \\
\hline \multicolumn{11}{|l|}{ Procedures at ED } \\
\hline Intubation & $18(21.2)$ & $5(5.1)$ & $8(17.0)$ & $117(3.4)$ & $7.7(4.5-13.4)$ & $<0.001$ & $1.5(0.6-3.9)$ & 0.348 & $5.9(2.7-12.9)$ & $<0.001$ \\
\hline Chest tube insertion & 9 (10.6) & $3(3.1)$ & $5(10.6)$ & $43(1.2)$ & $9.5(4.5-20.1)$ & $<0.001$ & $2.5(0.8-8.3)$ & 0.113 & $9.5(3.6-25.3)$ & $<0.001$ \\
\hline Blood transfusion & $21(24.7)$ & $4(4.1)$ & $15(31.9)$ & $92(2.6)$ & $12.1(7.1-20.7)$ & $<0.001$ & $1.6(0.6-4.4)$ & 0.383 & $17.3(9.1-33.0)$ & $<0.001$ \\
\hline
\end{tabular}


Table 3. Associated injuries of hospitalized trauma patients.

\begin{tabular}{|c|c|c|c|c|c|c|c|c|c|c|}
\hline \multirow{4}{*}{$\begin{array}{c}\text { Variables } \\
\begin{array}{c}\text { Head/Neck trauma } \\
\text { Neurologic deficit }\end{array}\end{array}$} & \multirow{2}{*}{$\begin{array}{c}\text { EMS RSI } \geqslant 1 \text { ED } \\
\text { RSS <1 } \\
n=85 \text { (II) }\end{array}$} & \multirow{2}{*}{$\begin{array}{c}\text { EMS RSI }<1 \text { ED } \\
\text { RSI } \geqslant 1 \\
n=98 \text { (III) }\end{array}$} & \multirow{2}{*}{$\begin{array}{c}\text { EMS RSI }<1 \text { ED } \\
\text { RSI }<1 \\
n=47 \text { (IV) }\end{array}$} & \multirow{2}{*}{$\begin{array}{c}\text { EMS RSI } \geqslant 1 \text { ED } \\
\text { RSI } \geqslant 1 \\
n=3485 \text { (I) }\end{array}$} & \multirow{2}{*}{\multicolumn{2}{|c|}{$\begin{array}{c}\text { OR }(95 \% C I) p \\
\text { II } v s . \text { I }\end{array}$}} & \multirow{2}{*}{\multicolumn{2}{|c|}{$\begin{array}{c}\text { OR }(95 \% C I) p \\
\text { III } v s . \mathrm{I}\end{array}$}} & \multirow{2}{*}{\multicolumn{2}{|c|}{$\begin{array}{c}\text { OR }(95 \% C I) p \\
\text { IV } v s . I\end{array}$}} \\
\hline & & & & & & & & & & \\
\hline & & & & & & & & & & \\
\hline & $\begin{array}{l}1(1.2) \\
10(11.8)\end{array}$ & $1(1.0)$ & $0(0.0)$ & $24(0.7)$ & $17(0.2-3.3)$ & 0.594 & $1.5(0.2-11.1)$ & 0.697 & $1.0(1.0-1.0)$ & 0.568 \\
\hline $\begin{array}{l}\text { Cranial fracture } \\
\text { Epidural hematoma }\end{array}$ & $\begin{array}{l}10 \\
5(5.9)\end{array}$ & $4(4.1)$ & $\begin{array}{l}1(2.1) \\
2(4.3)\end{array}$ & $173(5.0)$ & $1.2(0.5-3.0)$ & 0.701 & $0.8(0.4-1.9)$ & $\begin{array}{l}0.032 \\
0.691\end{array}$ & $0.3(0.0-2.0)$ & 0.168 \\
\hline Subdural hematoma & $10(11.8)$ & $7(7.1)$ & $4(8.5)$ & $319(9.2)$ & $1.3(0.7-2.6)$ & 0.411 & $0.8(0.4-1.7)$ & 0.495 & $0.9(0.3-2.6)$ & 0.879 \\
\hline Subarachnoid & $10(11.8)$ & $8(8.2)$ & $5(10.6)$ & $382(11.0)$ & $1.1(0.6-2.1)$ & 0.815 & $0.7(0.4-1.5)$ & 0.380 & $1.0(0.4-2.5)$ & 0.944 \\
\hline $\begin{array}{l}\text { nemorrnage } \\
\text { Intracerebral hematoma }\end{array}$ & $6(7.1)$ & $1(1.0)$ & $0(0.0)$ & $69(2.0)$ & $3.8(1.6-8.9)$ & 0.001 & $0.5(0.1-3.7)$ & 0.498 & & 0.330 \\
\hline Cerebral contusion & $5(5.9)$ & $8(8.2)$ & $1(2.1)$ & $173(5.0)$ & $1.2(0.5-3.0)$ & 0.701 & $1.7(0.8-3.6)$ & 0.154 & $0.4(0.1-3.0)$ & 0.372 \\
\hline $\begin{array}{l}\text { Cervical vertebral } \\
\text { fracture }\end{array}$ & $3(3.5)$ & $1(1.0)$ & $1(2.1)$ & $28(0.8)$ & $4.5(1.4-15.2)$ & 0.007 & $1.3(0.2-9.5)$ & 0.813 & $2.7(0.4-20.2)$ & 0.318 \\
\hline \multicolumn{11}{|l|}{ Maxillofacial trauma } \\
\hline Orbital fracture & $0(0.0)$ & $4(4.1)$ & $0(0.0)$ & $77(2.2)$ & - & 0.166 & $1.9(0.7-5.3)$ & 0.219 & & 0.303 \\
\hline Maxillary fracture & $0(0.0)$ & $1(1.0)$ & $1(2.1)$ & $54(1.5)$ & & 0.248 & $0.7(0.1-4.8)$ & 0.674 & $1.4(0.2-10.2)$ & 0.750 \\
\hline Mandibular fracture & $6(7.1)$ & $10(10.2)$ & $6(12.8)$ & $247(7.1)$ & $1.0(0.4-2.3)$ & 0.992 & $1.5(0.8-2.9)$ & 0.238 & $1.9(0.8-4.6)$ & 0.134 \\
\hline \multicolumn{11}{|l|}{$\begin{array}{l}\text { Nasal fracture } \\
\text { Thoracic trauma }\end{array}$} \\
\hline $\begin{array}{l}\text { Thoracict crauma } \\
\text { Rib fracture }\end{array}$ & $11(12.9)$ & $17(17.3)$ & $14(29.8)$ & $357(10.2)$ & $1.3(0.7-2.5)$ & 0.419 & $1.8(1.1-3.1)$ & 0.023 & $3.7(2.0-7.0)$ & $<0.001$ \\
\hline Hemothorax & $3(3.5)$ & & $4(8.5)$ & & $2.6(0.8-8.6)$ & 0.099 & $4.7(2.0-11.2)$ & $<0.001$ & $6.7(2.3-19.3)$ & $<0.001$ \\
\hline $\begin{array}{l}\text { Pneumothorax } \\
\text { Pnem }\end{array}$ & $4(4.7)$ & $2(2.0)$ & $\begin{array}{l}3(6.4) \\
3(10)\end{array}$ & $57(1.6)$ & $3.0(1.1-8.4)$ & 0.031 & $1.3(0.3-5.2)$ & 0.756 & $4.1(1.2-13.6)$ & 0.012 \\
\hline Hemopneumothorax & $5(5.9)$ & $2(2.0)$ & $6(12.8)$ & $35(1.0)$ & $6.2(2.4-16.1)$ & $<0.001$ & $2.1(0.5-8.7)$ & 0.317 & $14.4(5.8-36.2)$ & $<0.001$ \\
\hline Lung contusion & $4(4.7)$ & $0(0.0)$ & $0(0.0)$ & $43(1.2)$ & $4.0(1.4-11.3)$ & 0.006 & & 0.269 & & 0.444 \\
\hline $\begin{array}{l}\text { Abdominal trauma } \\
\text { Intra-abdominal injury }\end{array}$ & & & & & $22(08-61)$ & & $05(01-33)$ & & & \\
\hline $\begin{array}{l}\text { Intra-abdominal injury } \\
\text { Hepatic injury }\end{array}$ & $\begin{array}{c}4 \\
12(14.1)\end{array}$ & $\begin{array}{l}1(1.0) \\
5(5.1)\end{array}$ & $7(14.9)$ & $70(2.0)$ & $\begin{array}{l}2.2(0.8-6.1) \\
8.0(4.2-15.4)\end{array}$ & $<0.001$ & $\begin{array}{l}0.5(0.1-1.3) \\
2.6(1.0-6.7)\end{array}$ & $\begin{array}{l}0.426 \\
0.035\end{array}$ & $8.5(3.7-19.7)$ & $<0.001$ \\
\hline Splenic injury & $\begin{array}{l}4(4.7) \\
4(14)\end{array}$ & $2(2.0)$ & $4(8.5)$ & $28(0.8)$ & $6.1(2.1-17.8)$ & $<0.001$ & $2.6(0.6-11.0)$ & 0.185 & $11.5(3.9-34.2)$ & $<0.001$ \\
\hline Retroperitoneal injury & $2(2.4)$ & $0(0.0)$ & $0(0.0)$ & $5(0.1)$ & $16.8(3.2-87.7)$ & $<0.001$ & - & 0.707 & & 0.795 \\
\hline \multicolumn{11}{|l|}{$\begin{array}{c}\text { Renal injury } \\
\text { Extremity trauma }\end{array}$} \\
\hline Humeral fracture & & $5(5.1)$ & & $174(5.0)$ & $0.2(0.0-1.6)$ & 0.107 & $1.0(0.4-2.6)$ & 0.961 & $1.3(0.4-4.2)$ & 0.664 \\
\hline Radial fracture & $5(5.9)$ & $7(7.1)$ & $3(6.4)$ & $351(10.1)$ & $0.6(0.2-1.4)$ & 0.203 & $0.7(0.3-1.5)$ & 0.340 & $0.6(0.2-2.0)$ & 0.403 \\
\hline Ulnar fracture & $3(3.5)$ & $5(5.1)$ & $4(8.5)$ & $173(5.0)$ & $0.7(0.2-2.2)$ & 0.546 & $1.0(0.4-2.6)$ & 0.951 & $1.8(0.6-5.0)$ & 0.268 \\
\hline Pelvic fracture & $6(7.1)$ & $4(4.1)$ & $7(14.9)$ & $121(3.5)$ & $2.1(0.9-4.9)$ & 0.078 & $1.2(0.4-3.3)$ & 0.746 & $4.9(2.1-11.1)$ & $<0.001$ \\
\hline Femoral fracture & $14(16.5)$ & $14(14.3)$ & $8(17.0)$ & $393(11.3)$ & $1.6(0.9-2.8)$ & 0.137 & $1.3(0.7-2.3)$ & 0.355 & $1.6(0.8-3.5)$ & 0.218 \\
\hline Tibial fracture & $10(11.8)$ & $14(14.3)$ & $5(10.6)$ & $373(10.7)$ & $1.1(0.6-2.2)$ & 0.755 & $1.4(0.8-2.5)$ & 0.260 & $1.0(0.4-2.5)$ & 0.989 \\
\hline Fibular fracture & $5(5.9)$ & $10(10.2)$ & $3(6.4)$ & $222(6.4)$ & $0.9(0.4-2.3)$ & 0.856 & $1.7(0.9-3.3)$ & 0.128 & $1.0(0.3-3.3)$ & 0.997 \\
\hline
\end{tabular}

Table 4. Length of stay in the hospital and the intensive care unit.

\begin{tabular}{|c|c|c|c|c|c|c|c|c|c|c|}
\hline \multirow{2}{*}{ Variables } & \multirow{2}{*}{$\begin{array}{l}\text { EMS RSI } \geqslant 1 \text { ED } \\
\text { RSI }<1 n=85 \text { (II) }\end{array}$} & \multirow{2}{*}{$\begin{array}{l}\text { EMS RSI < } 1 \text { ED RSI } \\
\quad \geqslant 1 n=98 \text { (III) }\end{array}$} & \multirow{2}{*}{$\begin{array}{l}\text { EMS RSI }<1 \text { ED } \\
\text { RSI }<1 n=47 \text { (IV) }\end{array}$} & \multirow{2}{*}{$\begin{array}{c}\text { EMS RSI } \geqslant 1 \text { ED RSI } \\
\qquad \geqslant 1 n=3485 \text { (I) }\end{array}$} & \multirow{2}{*}{\multicolumn{2}{|c|}{$\begin{array}{c}O R(95 \% C I) p \\
\text { II vs. I }\end{array}$}} & \multicolumn{2}{|c|}{ OR $(95 \% C I) p$} & \multicolumn{2}{|c|}{ OR $(95 \% C I) p$} \\
\hline & & & & & & & & & & \\
\hline $\begin{array}{c}\text { Hospital LOS } \\
\text { days } \\
\text { ICU LOS }\end{array}$ & $15.2 \pm 14.5$ & $10.9 \pm 12.0$ & $16.9 \pm 16.2$ & $9.5 \pm 9.4$ & - & 0.001 & - & 0.156 & - & $<0.001$ \\
\hline $\begin{array}{l}\mathrm{n}(\%) \\
\text { days }\end{array}$ & $\begin{array}{c}39(45.9) \\
13.1 \pm 14.3\end{array}$ & $\begin{array}{l}19(19.4) \\
6.5 \pm 6.2\end{array}$ & $\begin{array}{c}24(51.1) \\
13.0 \pm 20.3\end{array}$ & $\begin{array}{l}575(16.5) \\
7.6 \pm 8.2\end{array}$ & $4.3(2.8-6.6)$ & $\begin{array}{l}<0.001 \\
0.296\end{array}$ & $1.2(0.7-2.0)$ & $\begin{array}{l}0.448 \\
0.397\end{array}$ & $5.3(3.0-9.4)$ & $\begin{array}{c}<0.001 \\
0.070\end{array}$ \\
\hline
\end{tabular}


Therefore, specific attention should be provided to group II patients because they have a higher AOR for mortality than patients in other groups. For example, group II patients had an AOR for mortality 5.8-times greater than that of group I patients while group IV patients had an AOR for mortality 1.1-times greater than group I patients. Group II patients are younger and sustained injuries to more regions (excluding the face) than group I patients. Considering that the mean transport time was short (less than $25 \mathrm{~min}$ ) in all groups, the quickly deteriorating hemodynamic condition upon arrival to the ED is an alarming sign for these relatively younger patients. In particular, these patients are more likely to have a severe injury to the thoracoabdominal areas. A higher OR was also found for sustaining pneumothorax (3.0-fold), hemopneumothorax (6.2-fold), a lung contusion (4.0-fold), hepatic injury (8.0-fold), splenic injury (6.1-fold), retroperitoneal injury (16.8-fold), and renal injury (6.4-fold) among group II patients when compared with group I patients. In addition, a higher OR for intubation (7.7-fold), chest tube insertion (9.5-fold), and blood transfusion (12.1-fold) was noted in group II patients as well.

Furthermore, group IV and group II patients had similar characteristics and a similar injury pattern. Group IV patients had a similarly high ISS as well as a higher OR for sustaining a rib fracture (3.7-fold), hemothorax (6.7-fold), pneumothorax (4.1-fold), hemopneumothorax (14.4-fold), an intra-abdominal injury (7.7-fold), hepatic injury (8.5-fold), splenic injury (11.5-fold), and pelvic fracture (4.9-fold) when compared to group I patients. In addition, a higher OR for intubation (5.9-fold), chest tube insertion (9.5-fold), and blood transfusion (17.3-fold) was also found. However, the AOR for mortality was only slightly significantly higher than that of group I patients (AOR: 1.1, 95\% CI: 1.1-1.1, $p<0.001$ ), even though hemodynamic instability was established at both the injury scene and in the ED. No strong evidence defining the optimal blood pressure level during active hemorrhagic shock has been documented in the literature $[25,26]$ and the optimal blood pressure level during resuscitation of a hemorrhagic shock patient is still debated. One study proposed administering a limited amount of fluids before bleeding control to maintain arterial pressure and minimize dilution of coagulation factors as well as complications from over fluid resuscitation [27]. For patients with trauma but without brain injury, European guidelines recommend a target SBP of 80-90 mmHg until major bleeding in the initial phase has been stopped [28]. Moreover, a policy of permissive hypotension with judicious fluid administration to maintain a mean arterial pressure in the $60-80 \mathrm{mmHg}$ range is advisable and appropriate [29-31]. Notably, the significantly higher incidence of hemothorax and pelvic fracture in group IV patients accompanied by massive blood loss had to be treated with blood or fluid transfusions as well as more advanced procedures (i.e., chest tube insertion or pressure garment compression, respectively). The higher mortality rate found in group II patients compared to group IV patients indicates that patients who have a stable hemodynamic condition at the injury scene but experience a rapid deterioration of their hemodynamic status require specific attention. Young patients who present with tachycardia and mild hypotension are in danger of compensatory mechanism failure and may slip into profound shock unless vigorous therapy is initiated [14]. Reliance on SBP alone may delay recognition of the shock state [14].

Traumatic injury remains the leading cause of death among people less than 44 years old and $40 \%$ of trauma-related deaths are caused by uncontrolled hemorrhagic shock or its sequelae (such as multiple organ failure) [32]. Significant, untapped opportunities for early recognition and treatment of critical illnesses may exist within the EMS population. Assessment and treatment of trauma patients upon arrival to the ED is essential in the presence of life-threatening injuries. Prospectively identifying patients that would benefit from trauma care is essential to the success of trauma systems. Although little is known about the ability of EMS personnel to accurately approximate the volume of blood loss in an out-of-hospital setting, previous studies have reported that EMS providers are not able to adequately estimate spilled blood volumes [33]. Following the advanced trauma life support paradigm, "keep algorithms simple", an RSI < 1 may serve as an alert for attending physicians in the ED. Given the circumstances in an ED (e.g., many patients waiting in crowded EDs for hours before a physician evaluation) [34], the timely recognition and rapid treatment of shock can be a difficult task. One of 
the major benefits of using the RSI for evaluation in the ED is that it can be used quickly when first responders arrive without requiring any additional equipment or cost. An RSI $<1$ can alert trauma surgeons to the need for early intervention and timely preparation upon the arrival of the patient. In particular, attention should be placed on those with an EMS RSI $\geqslant 1$ that deteriorates in the ED. Using the EMS RSI as an additional information may also help alert physicians to a thoracoabdominal injury with massive bleeding and help to identify patients with serious injuries who need upgraded higher level of intervention. This may help improve patient outcomes after a severe injury.

Our analysis has several limitations. First, the data were collected prospectively as part of the required trauma registry process, but our analyses were performed retrospectively and are thus subject to the limitations of all retrospective studies. Second, injured patients who did not survive until arrival at the hospital or who were discharged from the ED were not included in the sample, which could result in a selection bias. Third, age, hypertension, and $\beta$ - or calcium channel blockers weaken the association between the SI and mortality rate [23], and the impact of pre-existing comorbidities on the course of hospitalization and mortality was not included in the analysis and, thus, remains unclear. In addition, some missing data and the lack of available data regarding patient management, including type, volume, and speed of fluid resuscitation during transportation by EMS and in the ED, may result in a bias in the outcome. Finally, some important data, such as cost, treatment delays, and complications, were not evaluated and may have limited the outcome evaluation results.

\section{Conclusions}

This retrospective analysis spanning a five-year period showed that using an RSI $<1$ as a threshold to evaluate the hemodynamic condition of trauma patients at the injury scene and upon arrival to the ED provides valid information regarding the deteriorating outcomes of certain subgroups of patients in the ED setting. Particular attention and resources should be provided for patients with an EMS RSI $\geqslant 1$ that deteriorates to an RSI $<1$ upon arrival to the ED since a higher odds of mortality was found in these patients.

Acknowledgments: The research reported in this publication was supported by a grant from Chang Gung Memorial Hospital (CDRPG8C0031).

Author Contributions: Wei-Hung Lai and Cheng-Shyuan Rau wrote the manuscript, Shiun-Yuan Hsu carried out the data collection and analysis, Shao-Chun Wu and Pao-Jen Kuo involved in data analysis, Hsiao-Yun Hsieh proofread the manuscript, Yi-Chun Chen edited the tables and Ching-Hua Hsieh designed the entire study and contributed to the data analysis and interpretation. All authors have read and approved the final manuscript.

Conflicts of Interest: The authors declare no conflict of interest.

\section{Abbreviations}

The following abbreviations are used in this manuscript:

$\begin{array}{ll}\text { AOR } & \text { Adjusted odd ratio } \\ \text { AIS } & \text { Abbreviated injury scale } \\ \text { CI } & \text { Confidence interval } \\ \text { ED } & \text { Emergency department } \\ \text { EMS } & \text { Emergency medical services } \\ \text { GCS } & \text { Glasgow coma scale } \\ \text { HR } & \text { Heart rate } \\ \text { ICU } & \text { Intensive care unit } \\ \text { ISS } & \text { Injury severity score } \\ \text { LOS } & \text { Length of stay } \\ \text { NISS } & \text { New injury severity score } \\ \text { OR } & \text { Odds ratio } \\ \text { RSI } & \text { Reverse shock index } \\ \text { SI } & \text { Shock index } \\ \text { SBP } & \text { Systolic blood pressure } \\ \text { TRISS } & \text { Trauma and injury severity score }\end{array}$




\section{References}

1. Chan, L.; Bartfield, J.M.; Reilly, K.M. The significance of out-of-hospital hypotension in blunt trauma patients. Acad. Emerg. Med. 1997, 4, 785-788. [CrossRef] [PubMed]

2. Patton, K.; Funk, D.L.; McErlean, M.; Bartfield, J.M. Accuracy of estimation of external blood loss by EMS personnel. J. Trauma 2001, 50, 914-916. [CrossRef] [PubMed]

3. Holler, J.G.; Bech, C.N.; Henriksen, D.P.; Mikkelsen, S.; Pedersen, C.; Lassen, A.T. Nontraumatic hypotension and shock in the emergency department and the prehospital setting, prevalence, etiology, and mortality: A systematic review. PLoS ONE 2015, 10, e0119331. [CrossRef] [PubMed]

4. Victorino, G.P.; Battistella, F.D.; Wisner, D.H. Does tachycardia correlate with hypotension after trauma? J. Am. Coll. Surg. 2003, 196, 679-684. [CrossRef]

5. Brasel, K.J.; Guse, C.; Gentilello, L.M.; Nirula, R. Heart rate: Is it truly a vital sign? J. Trauma 2007, 62, 812-817. [CrossRef] [PubMed]

6. Havel, C.; Arrich, J.; Losert, H.; Gamper, G.; Mullner, M.; Herkner, H. Vasopressors for hypotensive shock. Cochrane Database Syst. Rev. 2011, 5. [CrossRef]

7. Andersen, G.O. Circulatory shock. N. Engl. J. Med. 2014, 370, 583. [PubMed]

8. Bruns, B.; Gentilello, L.; Elliott, A.; Shafi, S. Prehospital hypotension redefined. J. Trauma 2008, 65, $1217-1221$. [CrossRef] [PubMed]

9. Lalezarzadeh, F.; Wisniewski, P.; Huynh, K.; Loza, M.; Gnanadev, D. Evaluation of prehospital and emergency department systolic blood pressure as a predictor of in-hospital mortality. Am. Surg. 2009, 75, 1009-1014. [PubMed]

10. Hasler, R.M.; Nuesch, E.; Juni, P.; Bouamra, O.; Exadaktylos, A.K.; Lecky, F. Systolic blood pressure below $110 \mathrm{mmHg}$ is associated with increased mortality in penetrating major trauma patients: Multicentre cohort study. Resuscitation 2012, 83, 476-481. [CrossRef] [PubMed]

11. King, R.W.; Plewa, M.C.; Buderer, N.M.; Knotts, F.B. Shock index as a marker for significant injury in trauma patients. Acad. Emerg. Med. 1996, 3, 1041-1045. [CrossRef] [PubMed]

12. Rady, M.Y.; Smithline, H.A.; Blake, H.; Nowak, R.; Rivers, E. A comparison of the shock index and conventional vital signs to identify acute, critical illness in the emergency department. Ann. Emerg. Med. 1994, 24, 685-690. [CrossRef]

13. Cannon, C.M.; Braxton, C.C.; Kling-Smith, M.; Mahnken, J.D.; Carlton, E.; Moncure, M. Utility of the shock index in predicting mortality in traumatically injured patients. J. Trauma 2009, 67, 1426-1430. [CrossRef] [PubMed]

14. Gutierrez, G.; Reines, H.D.; Wulf-Gutierrez, M.E. Clinical review: Hemorrhagic shock. Crit. Care 2004, 8, 373-381. [CrossRef] [PubMed]

15. Mitra, B.; Fitzgerald, M.; Chan, J. The utility of a shock index $\geqslant 1$ as an indication for pre-hospital oxygen carrier administration in major trauma. Injury 2014, 45, 61-65. [CrossRef] [PubMed]

16. McNab, A.; Burns, B.; Bhullar, I.; Chesire, D.; Kerwin, A. An analysis of shock index as a correlate for outcomes in trauma by age group. Surgery 2013, 154, 384-387. [CrossRef] [PubMed]

17. DeMuro, J.P.; Simmons, S.; Jax, J.; Gianelli, S.M. Application of the Shock Index to the prediction of need for hemostasis intervention. Am. J. Emerg. Med. 2013, 31, 1260-1263. [CrossRef] [PubMed]

18. Rady, M.Y.; Rivers, E.P.; Martin, G.B.; Smithline, H.; Appelton, T.; Nowak, R.M. Continuous central venous oximetry and shock index in the emergency department: Use in the evaluation of clinical shock. Am. J. Emerg. Med. 1992, 10, 538-541. [CrossRef]

19. Mutschler, M.; Nienaber, U.; Munzberg, M.; Wolfl, C.; Schoechl, H.; Paffrath, T.; Bouillon, B.; Maegele, M. The Shock Index revisited-A fast guide to transfusion requirement? A retrospective analysis on 21,853 patients derived from the TraumaRegister DGU. Crit. Care 2013, 17, R172. [CrossRef] [PubMed]

20. Rady, M.Y.; Nightingale, P.; Little, R.A.; Edwards, J.D. Shock index: A re-evaluation in acute circulatory failure. Resuscitation 1992, 23, 227-234. [CrossRef]

21. Heffner, A.C.; Swords, D.S.; Nussbaum, M.L.; Kline, J.A.; Jones, A.E. Predictors of the complication of postintubation hypotension during emergency airway management. J. Crit. Care 2012, 27, 587-593. [CrossRef] [PubMed]

22. Edla, S.; Reisner, A.T.; Liu, J.; Convertino, V.A.; Carter, R., 3rd; Reifman, J. In reply to “Utility of shock index calculation in hemorrhagic trauma". Am. J. Emerg. Med. 2015, 33, 978-979. [CrossRef] [PubMed] 
23. Kristensen, A.K.; Holler, J.G.; Hallas, J.; Lassen, A.; Shapiro, N.I. Is shock index a valid predictor of mortality in emergency department patients with hypertension, diabetes, high age, or receipt of $\beta$ - or calcium channel blockers? Ann. Emerg. Med. 2016, 67, 106-113. [CrossRef] [PubMed]

24. McNab, A.; Burns, B.; Bhullar, I.; Chesire, D. Kerwin, A. A prehospital shock index for trauma correlates with measures of hospital resource use and mortality. Surgery 2012, 152, 473-476. [CrossRef] [PubMed]

25. Morrison, C.A.; Carrick, M.M.; Norman, M.A.; Scott, B.G.; Welsh, F.J.; Tsai, P.; Liscum, K.R.; Wall, M.J., Jr.; Mattox, K.L. Hypotensive resuscitation strategy reduces transfusion requirements and severe postoperative coagulopathy in trauma patients with hemorrhagic shock: Preliminary results of a randomized controlled trial. J. Trauma 2011, 70, 652-663. [CrossRef] [PubMed]

26. Kwan, I.; Bunn, F.; Chinnock, P.; Roberts, I. Timing and volume of fluid administration for patients with bleeding. Cochrane Database Syst. Rev. 2014, 3, Cd002245. [PubMed]

27. Harrois, A.; Hamada, S.R.; Duranteau, J. Fluid resuscitation and vasopressors in severe trauma patients. Curr. Opin. Crit. Care 2014, 20, 632-637. [CrossRef] [PubMed]

28. Spahn, D.R.; Bouillon, B.; Cerny, V.; Coats, T.J.; Duranteau, J.; Fernandez-Mondejar, E.; Filipescu, D.; Hunt, B.J.; Komadina, R.; Nardi, G.; et al. Management of bleeding and coagulopathy following major trauma: An updated European guideline. Crit. Care 2013, 17, R76. [CrossRef] [PubMed]

29. Hai, S.A. Permissive hypotensive resuscitation-An evolving concept in trauma. JPMA J. Pak. Med. Assoc. 2004, 54, 434-436. [PubMed]

30. Kreimeier, U.; Prueckner, S.; Peter, K. Permissive hypotension. Schweiz. Med. Wochenschr. 2000, 130, 1516-1524. [PubMed]

31. Smith, J.B.; Pittet, J.F.; Pierce, A. Hypotensive Resuscitation. Curr. Anesthesiol. Rep. 2014, 4, $209-215$. [CrossRef] [PubMed]

32. Dutton, R.P.; Stansbury, L.G.; Leone, S.; Kramer, E.; Hess, J.R.; Scalea, T.M. Trauma mortality in mature trauma systems: Are we doing better? An analysis of trauma mortality patterns, 1997-2008. J. Trauma 2010, 69, 620-626. [CrossRef] [PubMed]

33. Moscati, R.; Billittier, A.J.; Marshall, B.; Fincher, M.; Jehle, D.; Braen, G.R. Blood loss estimation by out-of-hospital emergency care providers. Prehosp. Emerg. Care 1999, 3, 239-242. [CrossRef] [PubMed]

34. Travers, D.A.; Waller, A.E.; Bowling, J.M.; Flowers, D.; Tintinalli, J. Five-level triage system more effective than three-level in tertiary emergency department. J. Emerg. Nurs. 2002, 28, 395-400. [CrossRef] [PubMed]

(C) 2016 by the authors; licensee MDPI, Basel, Switzerland. This article is an open access article distributed under the terms and conditions of the Creative Commons by Attribution (CC-BY) license (http://creativecommons.org/licenses/by/4.0/). 